\title{
Necesidades de personas en terapia de diálisis y sus cuidadores: Revisión integrativa
}

\author{
Diana Huérfano Martínez ${ }^{1}$, Dayana Álvarez Zambrano ${ }^{1}$, Estefani Arias Silvaํㅜ, Sonia Carreño Moreno² \\ ${ }^{1}$ Enfermera. Universidad Nacional de Colombia. Colombia \\ ${ }^{2}$ Enfermera. Magister en Enfermería. Doctora en Enfermería. Profesora. Universidad Nacional de Colombia. Colombia
}

\section{Resumen}

Introducción: Las personas con enfermedad renal crónica en tratamiento de diálisis y sus cuidadores tienen necesidades de diversa índole. Se requiere clarificarlas.

Objetivo: Integrar los hallazgos de la literatura disponible acerca de las necesidades de personas con enfermedad renal crónica en terapia de diálisis y de sus cuidadores.

Material y Método: Revisión integrativa de literatura en la que se incluyeron 37 estudios previa lectura crítica de la literatura. La extracción, sistematización e integración de hallazgos se realizó con técnica de análisis de contenido.

Resultados: Los hallazgos se agruparon en cinco temas: Necesitan promoción de la adaptación y afrontamiento, necesitan información y educación, necesitan seguimiento y reentrenamiento, necesitan promoción de su capacidad toma de decisiones y necesitan estrechar vínculos con el equipo de salud.

Conclusiones: La práctica del cuidado de la enfermería nefrológica requiere la construcción continua del vínculo con la persona con ERC y su cuidador, lo cual posibilita la identificación y satisfacción de sus necesidades. Es imperante la promoción de la autonomía y empoderamiento,

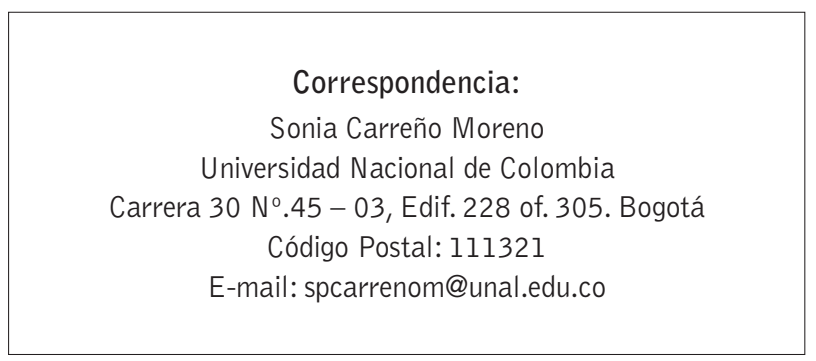

que apunte hacia la conciencia en la toma de decisiones y la gestión de la propia salud. La evidencia soporta que es necesaria la continuidad en la educación, el apoyo, el seguimiento y los vínculos.

PALABRAS CLAVE: insuficiencia renal crónica; diálisis renal; evaluación de necesidades; cuidadores; pacientes.

\section{Needs of people in dialysis therapy and their caregivers: integrative review}

\section{Abstract}

Introduction: People with chronic kidney disease on dialysis therapy and their caregivers have different needs, which require explanations.

Aim: To integrate the findings of available literature on the needs of people with chronic kidney disease on dialysis therapy and their caregivers.

Material and Method: Literature review. 37 studies were included after their critical review. The extraction, systematization and integration of findings was carried out using a content analysis technique.

Results: The findings were grouped into five themes: Need for promotion of adaptation and coping; Information and education requirement; Need for follow-up and retraining; Promotion of their decision-making capacity and Need for closer links with the health team.

Conclusions: The practice of nephrology nursing care requires the continuous construction of the link with chronic kidney disease patients and their caregiver, 
which permit to identify and satisfy their needs. It is imperative to promote autonomy and empowerment, pointing toward consciousness in decision-making and health management. The evidence supports the need for continuity in education, support, follow-up and links.

KEYWORDS: renal insufficiency chronic; renal dialysis; needs assessment; caregivers; patients.

\section{Introdución}

La persona con enfermedad renal crónica enfrenta varios retos en el manejo de su enfermedad, el más evidente de ellos, adaptarse a la terapia de diálisis y con ello, asumir las situaciones secundarias a ella, no sólo en términos de la dimensión física, sino en las consecuencias sociales, psicológicas y espirituales que se derivan de su situación de dependencial-3. En algunas ocasiones ante las disfunciones propias de la condición de cronicidad de la persona con ERC en terapia de diálisis, es necesario contar con un cuidador, quien es con frecuencia un familiar cercano que asume este rol y dentro de él, acciones complejas de cuidado como el acompañamiento, supervisión y en muchos casos ejecución de la terapia dialítica, administración de medicamentos, alimentación, asistencia en las actividades de la vida diaria, gestión del acceso a los servicios de salud, resolución de problemas y toma de decisiones ${ }^{4-7}$.

Bajo este panorama, son múltiples las necesidades que tanto las personas con ERC como sus cuidadores pueden presentar, no solo relacionadas con la información y entrenamiento en la terapia dialítica y acciones conexas, que de por sí, presentan un panorama desalentador derivado de la interacción superficial entre los profesionales, las personas con ERC y sus cuidadores ${ }^{8}$.

Consolidar lo reportado por la literatura en un marco global de necesidades de la persona con ERC y su cuidador, es una demanda prioritaria que permitirá expandir el conocimiento en el área y a su vez será una herramienta de rápida consulta para la toma de decisiones de cuidado de enfermería en los escenarios clínicos que expandirá las posibilidades de intervención.

El objetivo de esta revisión fue integrar los hallazgos de la literatura disponible acerca de las necesidades de personas con enfermedad renal crónica en terapia de diálisis y de sus cuidadores.

\section{Material y Método}

Se realizó una revisión integrativa de literatura científica ${ }^{9}$, para la cual se efectuaron los siguientes pasos:

1. Búsqueda de artículos en las bases de datos, Proquest, Sage, Willey online Library, Scopus, EMBASE, Medline, Scielo, Science Direct, PubMed, Cuiden, Academic Search Complete y LILACS. Para la búsqueda se usaron los descriptores necesidades, diálisis, ERC, hemodiálisis, diálisis peritoneal, información, psicosociales, prácticas, tratamiento, necesidades y sus equivalentes en inglés, combinándolos con la ayuda de operadores boléanos AND y OR ${ }^{10}$.

Fueron criterios de inclusión de los estudios: a) estar publicados en revistas indexadas entre los años 2010 a 2016, b) reportar hallazgos relacionados con las necesidades de los pacientes en tratamiento de diálisis o de sus cuidadores, c) evidenciar tanto el cumplimiento de los principios éticos para investigar con seres humanos como el respeto por la propiedad intelectual, y d) tener idioma original de publicación español, inglés o portugués.

Al realizar las ecuaciones de búsqueda se obtuvieron un total de 4.276 resultados, reduciéndose a 328 tras aplicar los filtros de año, de los cuales se preseleccionaron 126 tras encontrar en el título alguno de los descriptores.

2.Lectura crítica de estudios. En primer momento, se efectuó una lectura crítica de los resúmenes, siendo elegidos 36 estudios. Posterior a ello, los artículos seleccionados fueron leídos bajo los criterios propuestos por Bobenrieth ${ }^{11}$, con el fin de determinar su inclusión final en el estudio, cumpliendo todos con los criterios de inclusión y calidad.

3. Integración de los hallazgos. Los hallazgos fueron tratados con técnicas de análisis de contenido de la siguiente manera: a) fue determinado el objeto de análisis el cual fue las necesidades de personas en terapia de diálisis y sus cuidadores b) se estableció como regla de codificación la presencia del hallazgo importante dentro del texto, para este efecto se utilizó un sistema de codificación abierta inductiva c) a través de un proceso de clasificación de códigos y comparación, emergió un sistema de temas que agruparon códigos con algo en común entre sí12. 


\section{Resultados}

\section{Los resultados se agruparon en cinco temas emergentes.}

En la Tabla 1 y Tabla 2 pueden encontrar un resumen con el típo de artículos incluidos y la síntesis de los mismos.

Tabla 1. Tipo y cantidad de artículos seleccionados.

\begin{tabular}{|c|c|c|c|c|c|}
\hline & Aspecto & $\begin{array}{l}\text { Número de } \\
\text { artículos }\end{array}$ & & Aspecto & $\begin{array}{l}\text { Número de } \\
\text { artículos }\end{array}$ \\
\hline \multirow{7}{*}{$\begin{array}{l}\text { Abordaje } \\
\text { metodológico } \\
\text { del manuscrito }\end{array}$} & Cuantitativo descriptivo & 15 & \multirow{7}{*}{$\begin{array}{l}\text { Tipo de } \\
\text { necesidad } \\
\text { reportada }\end{array}$} & \multirow{2}{*}{$\begin{array}{l}\text { Promoción de la adaptación y } \\
\text { afrontamiento }\end{array}$} & \multirow{2}{*}{17} \\
\hline & Cuantitativo correlacional & 4 & & & \\
\hline & Ensayo clínico & 5 & & Información y educación & 13 \\
\hline & Estudio preexperimental & 1 & & Seguimiento y reentrenamiento & 6 \\
\hline & Cualitativo descriptivo & 8 & & $\begin{array}{l}\text { Promoción de capacidad de } \\
\text { tomar decisiones }\end{array}$ & 7 \\
\hline & Cualitativo fenomenólogico & 1 & & \multirow{2}{*}{$\begin{array}{l}\text { Estrechar vínculos con el equipo } \\
\text { de salud }\end{array}$} & \multirow[b]{2}{*}{5} \\
\hline & Revisión & 1 & & & \\
\hline
\end{tabular}

Tabla 2. Síntesis de los artículos seleccionados.

\begin{tabular}{|c|c|c|c|}
\hline Autor, año, país & Tipo de estudio & Muestra & Conclusiones \\
\hline $\begin{array}{l}\text { Castaño C, Hurtado L, } \\
\text { Perea D, Sánchez A. } \\
\text { 2013. Colombia }{ }^{13}\end{array}$ & $\begin{array}{l}\text { Descriptivo } \\
\text { transversal }\end{array}$ & $\begin{array}{l}86 \text { Pacientes con } \\
\text { ERC y sus cuidadores }\end{array}$ & $\begin{array}{l}\text { Los pacientes perciben baja calidad de vida } \\
\text { Los cuidadores no perciben altos niveles de sobrecarga }\end{array}$ \\
\hline $\begin{array}{l}\text { Martín RA. } 2014 . \\
\text { México }\end{array}$ & $\begin{array}{l}\text { Descriptivo } \\
\text { observacional }\end{array}$ & $\begin{array}{l}27 \text { personas con ERC } \\
\text { en diálisis }\end{array}$ & $\begin{array}{l}\text { Los pacientes temen sufrir un posible error médico } \\
\text { La mayoria tienen deficit de conocimientos sobre la patología }\end{array}$ \\
\hline $\begin{array}{l}\text { Arimatea Branco JM, } \\
\text { Luz Lisboa MT. } 2015 . \\
\text { Brasil }^{15}\end{array}$ & $\begin{array}{l}\text { Cualitativo } \\
\text { descriptivo }\end{array}$ & $\begin{array}{l}3 \text { grupos de encuentro } \\
\text { con } 9 \text { individuos }\end{array}$ & $\begin{array}{l}\text { Se requiere ayuda del personal de enfermeria para adaptación al } \\
\text { tratamiento Se debe reprensar en el modelo pedagógico }\end{array}$ \\
\hline $\begin{array}{l}\text { Chan R, Brooks R, } \\
\text { Gallagher M, Erlich J, } \\
\text { Snelling P, Chow J, et } \\
\text { al.2010. Australia }{ }^{16}\end{array}$ & $\begin{array}{l}\text { Estudio metodológico } \\
\text { de validación }\end{array}$ & $\begin{array}{l}475 \text { personas en } \\
\text { diálisis }\end{array}$ & Existe el concepto de pérdida en pacientes en diálisis \\
\hline $\begin{array}{l}\text { Suri RS, Larive B, Hall } \\
\text { Y, Kimmel PL, Kliger } \\
\text { AS, Levin N, et al. } \\
\text { 2014. Canadá17 }\end{array}$ & ECA & $\begin{array}{l}245 \text { cuidadores de } \\
\text { personas en diálisis }\end{array}$ & $\begin{array}{l}\text { La diálisis nocturna parece aumentar la percpeción de sobrecarga } \\
\text { del cuidador }\end{array}$ \\
\hline $\begin{array}{l}\text { Vázquez M, Bolaños } \\
\text { L, Alonso R. } 2011 . \\
\text { España }^{18}\end{array}$ & $\begin{array}{l}\text { Descriptivo } \\
\text { transversal }\end{array}$ & $\begin{array}{l}53 \text { pacientes en } \\
\text { diálisis peritoneal }\end{array}$ & $\begin{array}{l}\text { La depresión, ansiedad y alexitimia son predictores de la calidad } \\
\text { de vida relacionada con la salud }\end{array}$ \\
\hline $\begin{array}{l}\text { Abrahão SS, Ricas J, } \\
\text { Andrade DF, Pompeu } \\
\text { FC, Chamahum L, } \\
\text { Araújo TM, et al. } \\
\text { 2010. Brasil }{ }^{19}\end{array}$ & $\begin{array}{l}\text { Descriptivo } \\
\text { transversal }\end{array}$ & $\begin{array}{l}30 \text { pacientes } \\
\text { con ERC }\end{array}$ & El $18 \%$ de los pacientes tiene técnica inadecuada de diálisis \\
\hline $\begin{array}{l}\text { López D, Rodriguez } \\
\text { L, Carreño S, Cuenca } \\
\text { I, Chaparro L. } 2015 . \\
\text { Colombia }^{20}\end{array}$ & $\begin{array}{l}\text { Cualitativo } \\
\text { descriptivo }\end{array}$ & $\begin{array}{l}277 \text { relatos de cuida- } \\
\text { dores de personas en } \\
\text { dialisis peritoneal }\end{array}$ & $\begin{array}{l}\text { Los cuidadores adquieren nuevos conocimientos, interactuan con } \\
\text { otros, reciben apoyo, descansan y tienen oportunidades de mejora } \\
\text { que les permiten cambiar la perspectiva de vida, cuando participan } \\
\text { en grupos de apoyo }\end{array}$ \\
\hline
\end{tabular}




\section{[ Diana Huérfano Martínez, et al ]}

Necesidades de personas en terapia de diálisis y sus cuidadores: Revisión integrativa

\begin{tabular}{|c|c|c|c|}
\hline Autor, año, país & Tipo de estudio & Muestra & Conclusiones \\
\hline $\begin{array}{l}\text { Ulloa J, Rodríguez } \\
\text { M, Cruz M. } 2014 . \\
\text { Colombia }\end{array}$ & $\begin{array}{l}\text { Descriptivo } \\
\text { transversal }\end{array}$ & $\begin{array}{l}42 \text { pacientes } \\
\text { con ERC }\end{array}$ & $\begin{array}{l}\text { Las personas tienen afectadas las necesidades de eliminar bien } \\
\text { y evitar peligros }\end{array}$ \\
\hline $\begin{array}{l}\text { Arechabala MC, } \\
\text { Catoni MI, Palma } \\
\text { E, Barrios S. } 2011 . \\
\text { Chile }\end{array}$ & $\begin{array}{l}\text { Descriptivo } \\
\text { correlacional }\end{array}$ & $\begin{array}{l}162 \text { pacientes en } \\
\text { diálisis y sus } \\
\text { cuidadores }\end{array}$ & $\begin{array}{l}\text { Pacientes y cuidadores se sienten sobrecargados, hay una } \\
\text { asociación positiva entre sobrecarga y depresión en pacientes }\end{array}$ \\
\hline $\begin{array}{l}\text { Arenas Jiménez M, } \\
\text { Reig-Ferrer A, Álva- } \\
\text { rez-Ude F, Malek T, } \\
\text { Moledous A, Gil MT, } \\
\text { et al. 2011. España }{ }^{23}\end{array}$ & $\begin{array}{l}\text { Descriptivo } \\
\text { correlacional }\end{array}$ & $\begin{array}{l}239 \text { pacientes en } \\
\text { diálisis }\end{array}$ & $\begin{array}{l}\text { Tener una personalidad pesimista se asocia con ingresos } \\
\text { hospitalarios } \\
\text { Pacientes con personalidad optimista tienen mejor salud } \\
\text { percibida }\end{array}$ \\
\hline $\begin{array}{l}\text { Abrahão SS, Ricas J, } \\
\text { Andrade DF, Pompeu } \\
\text { FC, Chamahum L, } \\
\text { Araújo TM, et al. } \\
\text { 2010. Brasil }{ }^{24}\end{array}$ & $\begin{array}{l}\text { Cualitativo } \\
\text { descriptivo }\end{array}$ & $\begin{array}{l}30 \text { pacientes con } \\
\text { ERC }\end{array}$ & $\begin{array}{l}\text { Los pacientes se quejan por las limitaciones que la diálisis trae } \\
\text { para su vida y la de su cuidador }\end{array}$ \\
\hline $\begin{array}{l}\text { Sadala MLA, Bruzos } \\
\text { GA de S, Pereira ER, } \\
\text { Bucuvic EM. } 2012 . \\
\text { Brasil }^{25}\end{array}$ & $\begin{array}{l}\text { Cualitativo } \\
\text { fenomenológico }\end{array}$ & $\begin{array}{l}19 \text { pacientes } \\
\text { en diálisis }\end{array}$ & $\begin{array}{l}\text { Los pacientes expresan cambios drásticos en su vida, los más } \\
\text { significativos fueron la angustia, el dolor fisico y las limitaciones, } \\
\text { además del futuro incierto }\end{array}$ \\
\hline $\begin{array}{l}\text { Villarreal-Ríos E, } \\
\text { Cárdenas-Maldonado } \\
\text { C, Vargas-Daza ER, } \\
\text { Galicia-Rodríguez L, } \\
\text { Martínez-González L, } \\
\text { Baca-Baca R. 2014. } \\
\text { Brasil'27 }\end{array}$ & $\begin{array}{l}\text { Cuantitativo } \\
\text { descriptivo }\end{array}$ & $\begin{array}{l}151 \text { pacientes en } \\
\text { diálisis }\end{array}$ & $\begin{array}{l}\text { La diálisis significa un alto costo para el sistema y la familia. Es } \\
\text { más costoefectiva la diálisis peritoneal }\end{array}$ \\
\hline $\begin{array}{l}\text { Julián-Mauro JC, Mo- } \\
\text { linuevo-Tobalina JÁ, } \\
\text { Sánchez-González JC. } \\
\text { 2012. España }{ }^{28}\end{array}$ & $\begin{array}{l}\text { Cuantitativo } \\
\text { descriptivo }\end{array}$ & $\begin{array}{l}243 \text { pacientes en } \\
\text { diálisis }\end{array}$ & $\begin{array}{l}\text { La diálisis es un importante factor de incapacidad laboral. La } \\
\text { hemodiálisis es un factor más fuerte que la diálisis peritoneal }\end{array}$ \\
\hline $\begin{array}{l}\text { Kiliś-Pstrusińska } \\
\text { K, Wasilewska A, } \\
\text { Medyńska A, Bałasz- } \\
\text { Chmielewska I, Grenda } \\
\text { R, Kluska-Jóźwiak A, } \\
\text { et al. 2013. Polonia }{ }^{29}\end{array}$ & $\begin{array}{l}\text { Cuantitativo } \\
\text { descriptivo }\end{array}$ & $\begin{array}{l}41 \text { pacientes y sus } \\
\text { cuidadores }\end{array}$ & $\begin{array}{l}\text { Las familias requieren apoyo financiero, apoyo emocional } \\
\text { y apoyo para la autorrealización } \\
\text { Más de la mitad tienen alterada la función mental }\end{array}$ \\
\hline $\begin{array}{l}\text { Pastor J, Julián } \\
\text { J.2010. España }{ }^{30}\end{array}$ & $\begin{array}{l}\text { Cuantitativo } \\
\text { descriptivo }\end{array}$ & 202 pacientes & $\begin{array}{l}\text { La mayoria de pacientes no tienen suficientes conocimientos sobre } \\
\text { la terapia, esto se dificulta aún más en pacientes mayores }\end{array}$ \\
\hline $\begin{array}{l}\text { Sánchez González JC, } \\
\text { Martínez Martínez C, } \\
\text { Bethencourt Fernández } \\
\text { D, Pablos López M. } \\
\text { 2015. España }{ }^{31}\end{array}$ & $\begin{array}{l}\text { Cuantitativo } \\
\text { descriptivo }\end{array}$ & $\begin{array}{l}32 \text { pacientes en } \\
\text { hemodiálisis }\end{array}$ & $\begin{array}{l}\text { Los pacientes tienen conocimientos medios altos frente al manejo } \\
\text { de la enfermedad y cuidados }\end{array}$ \\
\hline $\begin{array}{l}\text { Teresa de las M, } \\
\text { Mayoral H, Rincón CM. } \\
\text { 2015. España }{ }^{32}\end{array}$ & $\begin{array}{l}\text { Cuantitativo } \\
\text { descriptivo }\end{array}$ & $\begin{array}{l}42 \text { pacientes en } \\
\text { diálisis }\end{array}$ & $\begin{array}{l}\text { Hay falta de adherencia a las pautas de dieta en paiente } \\
\text { en diálisis }\end{array}$ \\
\hline
\end{tabular}


[ Diana Huérfano Martínez, et al ]

Necesidades de personas en terapia de diálisis y sus cuidadores: Revisión integrativa

\begin{tabular}{|c|c|c|c|}
\hline Autor, año, país & Tipo de estudio & Muestra & Conclusiones \\
\hline $\begin{array}{l}\text { Peláez B, Fernández } \\
\text { M, Núñez M, González } \\
\text { I, Méndez A, Quintana } \\
\text { A. 2013. España }{ }^{33}\end{array}$ & $\begin{array}{l}\text { Cuantitativo } \\
\text { descriptivo }\end{array}$ & $\begin{array}{l}62 \text { pacientes } \\
\text { en diálisis }\end{array}$ & $\begin{array}{l}\text { El tener un programa de reentrenamiento periódico y } \\
\text { personalizado favorece la formación para el cuidado } \\
\text { de los pacientes }\end{array}$ \\
\hline $\begin{array}{l}\text { Jiménez E, Meléndez } \\
\text { M, Meza E, Agramón } \\
\text { K, Pereyra M, } \\
\text { Martínez N. } 2011 . \\
\text { México }^{34}\end{array}$ & Pre- experimental & $\begin{array}{l}95 \text { pacientes } \\
\text { en diálisis }\end{array}$ & $\begin{array}{l}\text { La educación favorece el conocimiento acerca de la diálisis } \\
\text { y la toma de decisiones frente a la misma }\end{array}$ \\
\hline $\begin{array}{l}\text { Carrillo Algarra AJ. } \\
\text { 2015. Colombia35 }\end{array}$ & $\begin{array}{l}\text { Cuantitativo } \\
\text { descriptivo }\end{array}$ & $\begin{array}{l}279 \text { pacientes en } \\
\text { diálisis peritoneal }\end{array}$ & $\begin{array}{l}\text { La mayoria de pacientes tiene una capacidad de agencia } \\
\text { de autocuidado alta }\end{array}$ \\
\hline $\begin{array}{l}\text { Neu AM, Richardson } \\
\text { T, Lawlor J, Stuart J, } \\
\text { Newland J, McAfee N, } \\
\text { et al. 2016. USA }\end{array}$ & $\begin{array}{l}\text { Cuantitativo } \\
\text { correlacional }\end{array}$ & $\begin{array}{l}644 \text { pacientes } \\
\text { en diálisis }\end{array}$ & $\begin{array}{l}\text { La estandarización de la atención, mejora los resultados en el } \\
\text { cumplimiento de prácticas de cuidado y disminuyó la incidencia } \\
\text { de peritonitis }\end{array}$ \\
\hline $\begin{array}{l}\text { Gunasekara WDVN, } \\
\text { Ng K-H, Chan Y-H, } \\
\text { Aragon E, Foong P-P, } \\
\text { Lau Y-W, et al. 2010. } \\
\text { Singapore }\end{array}$ & $\begin{array}{l}\text { Cuantitativo } \\
\text { correlacional }\end{array}$ & $\begin{array}{l}\text { Datos de incidencia } \\
\text { de peritonitis entre } \\
1988 \text { y } 2008\end{array}$ & $\begin{array}{l}\text { El establecimiento de un equipo especializado de enfermería } \\
\text { mejora los resultados de la terapia, disminuyendo el ìndice de } \\
\text { peritonitis }\end{array}$ \\
\hline $\begin{array}{l}\text { Bordin G, Casati M, } \\
\text { Sicolo N, Zuccherato } \\
\text { N, Eduati V. } 2007 . \\
\text { Italia }\end{array}$ & $\begin{array}{l}\text { Cuantitativo } \\
\text { observacional }\end{array}$ & $\begin{array}{l}150 \text { centros de } \\
\text { diálisis públicos }\end{array}$ & $\begin{array}{l}\text { Los programas de educación que incluyen seguimiento } \\
\text { disminuyen las tasas de peritonitis }\end{array}$ \\
\hline $\begin{array}{l}\text { Gardulf A, Palsson } \\
\text { M, Nicolay U. } 2011 . \\
\text { Suecia }^{39}\end{array}$ & Pre experimental & $\begin{array}{l}43 \text { pacientes } \\
\text { en diálisis }\end{array}$ & $\begin{array}{l}\text { Los programas esducativos estructurados que incluyan el } \\
\text { equilibrio en la ingesta de calcio y fosfato aunmenta el } \\
\text { conocimiento sobre este tema en pacientes y tiene efecto en la } \\
\text { disminución a largo plazo de niveles plasmáticos de fosfato }\end{array}$ \\
\hline $\begin{array}{l}\text { Machowska A, Alscher } \\
\text { MD, Reddy Vanga S, } \\
\text { Koch M, Aarup M, } \\
\text { Qureshi A, et al. } \\
\text { 2016. Suecia }{ }^{40}\end{array}$ & $\begin{array}{l}\text { Cuantitativo } \\
\text { correlacional }\end{array}$ & $\begin{array}{l}270 \text { pacientes } \\
\text { en diálisis }\end{array}$ & $\begin{array}{l}\text { La educación y apoyo a las decisiones permite a los pacientes } \\
\text { comprender y elegir la modalidad de diálisis }\end{array}$ \\
\hline $\begin{array}{l}\text { Robinski M, Mau W, } \\
\text { Wienke A, Girndt M. } \\
\text { 2016. Alemania }{ }^{41}\end{array}$ & $\begin{array}{l}\text { Cuantitativo } \\
\text { comparativo }\end{array}$ & $\begin{array}{l}780 \text { pacientes } \\
\text { en diálisis }\end{array}$ & $\begin{array}{l}\text { Los pacientes en diálisis peritoneal valoraron mejor la toma } \\
\text { de decisiones compartida que los pacientes en hemodiálisis }\end{array}$ \\
\hline $\begin{array}{l}\text { Cohen LM, Ruthazer } \\
\text { R, Moss AH, Germain } \\
\text { MJ. 2010. USA }\end{array}$ & $\begin{array}{l}\text { Cuantitativo } \\
\text { correlacional }\end{array}$ & $\begin{array}{l}512 \text { pacientes en } \\
\text { hemodiálisis }\end{array}$ & $\begin{array}{l}\text { La edad avanzada, demencia, enfermedad vascular periférica, } \\
\text { albúmina disminuida se asociaron con la mortalidad }\end{array}$ \\
\hline Moss AH. $2010^{43}$ & $\begin{array}{l}\text { Declaración } \\
\text { de expertos }\end{array}$ & NA & $\begin{array}{l}\text { Es clave promover la toma de decisiones compartida y más } \\
\text { informada }\end{array}$ \\
\hline $\begin{array}{l}\text { Janssen DJA, Spruit } \\
\text { MA, Schols JMGA, } \\
\text { van der Sande FM, } \\
\text { Frenken LA, Wouters } \\
\text { EFM. 2013. Paises } \\
\text { Bajos }^{44}\end{array}$ & $\begin{array}{l}\text { Cuantitativo } \\
\text { observacional }\end{array}$ & $\begin{array}{l}80 \text { pacientes } \\
\text { en diálisis }\end{array}$ & $\begin{array}{l}\text { Los pacientes necesitan recibir informaciòn sobre la carga de la } \\
\text { enfermedd, el pronostico. Debe mejorar la comunicaciòn con el } \\
\text { paciente }\end{array}$ \\
\hline
\end{tabular}




\begin{tabular}{l|l|l|l|}
\hline $\begin{array}{l}\text { Autor, año, país } \\
\text { De Rosenroll A, } \\
\text { Higuchi KS, Dutton } \\
\text { KS, Murray MA, } \\
\begin{array}{l}\text { Stacey D. 2013. } \\
\text { Canadá45 }\end{array}\end{array}$ & Tipo de estudio & Muestra & Conclusiones \\
\hline $\begin{array}{l}\text { Allen D, Wainwright } \\
\text { M, Hutchinson T. 2011. } \\
\text { Canadá46 }\end{array}$ & $\begin{array}{l}\text { Cualitativo descriptivo } \\
\text { etnográfico }\end{array}$ & $\begin{array}{l}10 \text { cuidadores } \\
\text { familiares de } \\
\text { pacientes en diálisis }\end{array}$ & $\begin{array}{l}\text { Los roles de los cuidadores incluyeron la presencia, el cuidar, } \\
\text { acompañar, compartir opiniones y preferencias }\end{array}$ \\
$\begin{array}{l}\text { Wemodiálisis } \\
\text { Walker RC, Howard } \\
\text { K, Morton RL, Palmer } \\
\text { SC, Marshall MR, } \\
\text { Tong A. 2016. Nueva } \\
\text { Zelanda47 }\end{array}$ & $\begin{array}{l}\text { Cualitativo } \\
\text { descriptivo }\end{array}$ & $\begin{array}{l}\text { Las personas encuentran interacciones negativas con el personal } \\
\text { de salud relacionadas con el cuidado fraccionado de la persona, } \\
\text { una lucha de conocimientos del paciente y el equipo de salud } \\
\text { y falta de decisiones compartida }\end{array}$ \\
\hline
\end{tabular}

\section{Necesitan promoción de la adaptación y afronta- miento}

El proceso de afrontamiento y adaptación en la persona con ERC requiere ser promovido por los profesionales de salud, en particular enfermería. Para ello se necesita apoyo social, el cual se convierte en un modulador de la incertidumbre, desgaste físico, dependencia y cambios en el estilo de vida, los cuales son factores condicionantes del proceso de adaptación ${ }^{13}$. Se ha demostrado que el apoyo social modula los índices de mortalidad ${ }^{14}$, aumenta de la percepción de calidad de vida ${ }^{15}$, disminuye la depresión ${ }^{16}$, mejora la adherencia al tratamiento y la aceptación de la enfermedad ${ }^{17}$, mejora la expresión de sentimientos ${ }^{18}$. En particular, el apoyo recibido de parte del equipo de salud, se relaciona con el aumento de la seguridad y autoestima y el favorecimiento en el proceso de toma de decisiones ${ }^{15}$, el cual sumado al apoyo familiar ha mostrado relacionarse con el éxito de la terapia de diálisis ${ }^{19}$.

En el caso de los cuidadores familiares, el apoyo les permite reconocer la importancia de su rol, compartir experiencias con otros en donde hay espacios para descansar, lo que se convierte en un factor de aumenta su energía e intención de mantenerse en su rol ${ }^{20}$.

Reformular la perspectiva de la situación también es un aspecto clave para el afrontamiento y adaptación. Para las personas con ERC con frecuencia el panorama es hostil, pues se enfrentan a situaciones como la carga económica, carencia de apoyo ${ }^{6}$, afectación laboral, limitación económica, ruptura familiar ${ }^{15}$, deterioro de las relaciones de pareja, restricciones físicas, exigencias de la terapia de diálisi ${ }^{21}$, dependencia de un cuidador $^{17}$, ansiedad ${ }^{21}$, depresión ${ }^{22}$, disminución de su calidad de vida ${ }^{6,13}$ y un consecuente aumento del riesgo de ingresos hospitalarios ${ }^{23}$ y de mortalidad ${ }^{22}$.

Los cuidadores por su parte, presentan síntomas depresivos, sentimientos de hostilidad ${ }^{22}$, temor ante lo desconocido, autoabandono ${ }^{6}$, estrés, cansancio, responsabilidades y actividades demandantes, alteraciones familiares ${ }^{24}$ y dependencia de los profesionales de salud ${ }^{25}$.

Favorecer la independencia ${ }^{15}$, ampliar las expectativas de éxito del tratamiento, basadas en conocimientos útiles y realistas, promover el bienestar físico y emocional del paciente ${ }^{16,26}$, priorizar si es posible la terapia de diálisis peritoneal como la alternativa más costo efectiva y con menor impacto en la independencia del paciente y cuidador ${ }^{27,28}$, proporcionar apoyo ${ }^{29}$, potenciar el autocuidado ${ }^{24}$, son intervenciones que permiten reconstruir un escenario menos hostil para la persona con ERC y su cuidador ${ }^{20}$, lo que redunda en una perspectiva real de la situación y en últimas mejora su capacidad de afrontamiento y adaptación.

\section{Necesitan información y educación}

La persona con ERC y su cuidador tienen variadas necesidades de educación, entre ellas, información sobre la enfermedad, opciones de tratamiento con sus respectivas ventajas e inconvenientes, espacios para resolución de dudas ${ }^{30}$, higiene de manos, elementos requeridos para la terapia ${ }^{24,31}$, ingesta de líquidos y potasio ${ }^{32}$. Asegurar la formación responsable sobre la técnica de diálisis es primordial para evitar riesgos y asegurar la calidad, aspecto que se optimiza a través de la construcción de vínculos entre la persona con ERC, su cuidador y el equipo de salud ${ }^{19,26}$. 
El proceso educativo no debe limitarse únicamente a la provisión de información, sino evaluarse en indicadores de resultado, entre los cuales son destacables el conocimiento de la terapia, el cumplimiento de la misma, el control de síntomas y el control de riesgos; en donde realizar un abordaje individualizado es una estrategia eficaz para lograr dichos indicadores ${ }^{33}$. Una adecuada estrategia educativa, mejora la adherencia al tratamiento, disminuye la incertidumbre y mejora los indicadores de resultado ${ }^{34}$.

Además es recomendable tratar temas como la actividad física, sueño, alimentación, higiene, recreación, interacción social, confort y actividad sexual6,13,20,35, con lo que se promoverá la calidad de vida.

\section{Necesitan seguimiento y reentrenamiento}

Con frecuencia la intensidad de la intervención de enfermería se concentra al inicio de la terapia de diálisis, pues se parte del supuesto de que la persona con ERC y su cuidador no cuenta con los conocimientos suficientes, sin embargo, a pesar de que con el paso del tiempo y la experiencia la persona con ERC y su cuidador adquieren mayor competencia para el cuidado, se requiere un proceso continuo de seguimiento y reentrenamiento ${ }^{33}$.

Se ha evidenciado que con el paso del tiempo y debido a la complejidad del tratamiento, se modifican inconscientemente las pautas de actuación, lo que puede generar riesgos en las terapias y los cuidados conexos 33 ; además en ocasiones, cuando se cree tener experticia sobre los cuidados, se suelen asumir actitudes de riesgo, indiscreciones y desafíos al tratamient $0^{35}$.

El contar con un programa formal de seguimiento y reentrenamiento ha demostrado en niños con terapia de diálisis peritoneal, disminución de peritonitis y de cumplimiento de prácticas de cuidado. Aspectos clave del seguimiento son las evaluaciones postentrenamiento, las visitas domiciliarias, la supervisión de un profesional de salud y una frecuencia mínima de seguimiento de 6 meses $^{36,37}$. De la misma manera, a nivel general se ha demostrado una correlación entre las visitas domiciliarias y el reentrenamiento con bajas tasas de peritonitis ${ }^{38}$.

Por lo anterior, es conveniente establecer un programa de reentrenamientos periódicos y personalizados ${ }^{33}$, que incluya el seguimiento por parte de un equipo de salud en el que la gestión de enfermería es central y aspectos como la atención individualizada, permiten su humanización ${ }^{39}$.

\section{Necesitan promoción de su capacidad toma de de- cisiones}

La toma de decisiones como necesidad de una persona con ERC se ha abordado de manera más amplia ante la necesidad de elegir la terapia dialítica de preferencia. El recibir información y tener la posibilidad de elegir la modalidad de diálisis se ha elevado a derecho de la persona ${ }^{30} \mathrm{e}$ incluso se ha documentado que el inicio no deseado de la terapia, conduce a peores resultados clínicos en términos de aumento de la morbimortalidad ${ }^{40}$.

Se ha observado un proceso de auto renuncia al derecho de decidir, pues se desconocen las consecuencias de las decisiones y el temor asociado a ello, hace que de manera tácita, este proceso quede en manos del médico tratante o el equipo de salud encargado ${ }^{30}$. Aunque las personas con ERC en terapia de diálisis y sus cuidadores desean independencia, en particular, no estar sujetos a decisiones médicas, requieren apoyo del equipo de salud, para la toma de decisiones compartidas. Dicho proceso, entendido como aquel en el que hay un asesoramiento participativo, ha demostrado mejorar la capacidad de toma de decisiones y la satisfacción con el tratamiento entre los pacientes ${ }^{41}$.

En la asesoría participativa, es importante conversar acerca del pronóstico de la persona ${ }^{42,43}$, las esperanzas, los temores y enfatizar en que a pesar de que el tratamiento es requerido, es importante que la decisión se tome, esto con el fin de hacer un tratamiento más consciente y que el cuidado venidero también sea producto de una decisión personal, lo que dará más consistencia a las acciones; además, es necesario que el asesoramiento sea continuo y se centre en la experiencia vivida de la persona con ERC y sus cuidadores ${ }^{44}$.

La toma de decisiones compartida, favorece el vínculo de la díada persona con ERC-cuidador, proceso en el que el apoyo del cuidador, hace que se amplíe la perspectiva positiva, el acompañamiento, el aprendizaje conjunto y el compartir opiniones y valores, lo que redunda en mejores resultados para los implicados ${ }^{45}$.

Necesitan estrechar vínculos con el equipo de salud La intensa interacción entre el equipo de salud, la persona con ERC y sus cuidadores, hacen que se creen vínculos estrechos, pues se vive en un entorno de cuidado que permite el mantenimiento de una función vital, como lo es la eliminación ${ }^{13,25,33}$. 
La limitaciones relacionadas con el sistema de salud y el aumento de pacientes en las unidades de hemodiálisis, hace que cada vez más las relaciones se vayan deteriorando, factor que es determinante en la adherencia al tratamiento. Llama la atención las manifestaciones de personas que aseguran haber pasado de un sistema compasivo y cariñoso a uno adverso en donde hay una lucha entre personas con ERC, sus cuidadores y los profesionales de salud. La lucha se agudiza por la poca o nula interacción, pues no hay lugar a comunicación alguna, ya que todo el tiempo los profesionales tienen prisa. También se describen batallas, en las cuales las personas que son ya expertas en el manejo de su enfermedad, intentan opinar sobre su manejo terapéutico y las mejores opciones acorde con su experiencia particular, recibiendo inadecuadas devoluciones por parte del equipo de salud, en su percepción, por considerar que ellos tienen la autoridad, situación que limita la participación en la toma de decisiones y que afecta la sensación de autonomía, sensación de control y bienestar ${ }^{46}$.

En el entorno domiciliario, las personas con ERC refieren relaciones con los profesionales de salud que se caracterizan por la unidireccionalidad, en términos del poder que tiene el profesional, por ser el poseedor de la información y en muchos casos de la decisiones no solo sobre la terapéutica sino sobre la vida de la persona ${ }^{47}$.

Las personas con ERC y sus cuidadores requieren estrechar sus vínculos con el equipo de salud, desarrollar una relación de confianza en la que se sientan importantes, considerados y empoderados $13,25,33,46,47$.

El profesional de salud requiere recibir entrenamiento que le permita dimensionar la importancia de estas relaciones para el éxito del tratamiento $0^{46,47}$.

\section{Discusión}

Esta revisión presenta una perspectiva amplificadora de las necesidades de la persona con ERC en terapia de diálisis y su cuidador familiar. Con frecuencia, se tiende a realizar una valoración reactiva de dichas necesidades en la que prima el mantenimiento de funciones vitales biológicas que dependen de un adecuado proceso de eliminación renal ${ }^{21}$, lo que redunda en la excesiva instrumentalización de los cuidados que se brindan y en consecuencia la deprivación del componente humano de los mismos ${ }^{46,47}$.
Es indiscutible que la persona con ERC en terapia de diálisis y su cuidador requieren información, educación, entrenamiento, seguimiento y reentrenamiento, necesidades que han sido ampliamente documentadas y su efecto ha sido probado en el éxito de la terapia dialítica y en la prevención de riesgos y complicaciones asociadas a ésta ${ }^{6,13,19,20,30-38}$. Sin embargo, en el componente intangible de las necesidades, es evidente que no solo la terapia dialítica es la que mantiene la vida de la persona con ERC, sino también lo es su capacidad de afrontar y adaptarse a los cambios que trae con sigo la enfermedad, ante lo cual, mantener su independencia, su autonomía y capacidad de tomar de decisiones, hace que la lucha tenga sentido y se mantenga la motivación y sensación de control. El vínculo que se establece con los profesionales de salud en la experiencia de vivir con ERC y reemplazar la eliminación renal por una terapia dialítica, es fundamental para potenciar la capacidad de afrontamiento de la persona con ERC y su cuidador ${ }^{13-19,30,40-47}$. La experiencia de vida de la persona con ERC y su cuidador, la forma como se construyen el vínculo entre sí y con el equipo de salud, son fenómenos que merecen ser ampliados en estudios futuros, además de su relación con el éxito en la terapia dialítica y el control de riesgos y complicaciones asociados a ésta. Ampliar la indagación acerca de las causas de las relaciones adversas entre el equipo de salud con los pacientes y cuidadores, además de diseñar intervenciones para mejorar dichas relaciones, también son aspectos que requieren ser estudiados.

Lo hallazgos de esta revisión son un insumo para la práctica del cuidado a la persona con ERC y su cuidador, pues se confirma que acciones como la provisión de información, la educación, el entrenamiento y seguimiento son claves, pero también se exhorta a los profesionales de salud a dedicar más tiempo e interés a la valoración de necesidades intangibles, entre las que se destacan el apoyo, el ánimo, la escucha, la autonomía, la toma de decisiones y las sanas relaciones.

Por último, se evidencia que se requiere una ampliación de los roles de los profesionales de salud en el cuidado de las personas con ERC en diálisis y sus cuidadores, que mantengan lo asistencial y educativo y trasciendan al rol humanizador ${ }^{48}$. 


\section{Conclusiones}

La práctica del cuidado de la enfermería nefrológica requiere la construcción continua del vínculo con la persona con ERC y su cuidador, lo cual posibilita la identificación y satisfacción de sus necesidades. Es imperante la promoción de la autonomía y empoderamiento, que apunte hacia la conciencia en la toma de decisiones y la gestión de la propia salud. La evidencia soporta que es necesaria la continuidad en la educación, el apoyo, el seguimiento y los vínculos.

El autor declara que no hay conflicto de interés.

Recibido: 5 enero 2017

Revisado: 17 agosto 2017

Modificado: 22 enero 2018

Aceptado: 16 febrero 2018

\section{Bibliografía}

1. Ruiz de Alegría-Fernández de Retana B, Basabe-Barañano N, Saracho-Rotaeche R. El afrontamiento como predictor de la calidad de vida en diálisis: un estudio longitudinal y multicéntrico. Nefrología. 2013;33(3):342-54.

2. Andreu Periz L, Moreno Arroyo C, Julve Ibañéz M. Valoración de pacientes tratados con hemodiálisis según la Ley de Promoción de la Autonomía Personal y Atención a las personas en situación de dependencia. Rev Soc Esp Enferm Nefrol 2010;13(4):252-57.

3. Camps Ballester E, Andreu Periz L, Colomer Codinachs $M$, Claramunt Fonts $L$, Pasaron Alonso M. Valoración del grado de autonomía funcional de pacientes renales crónicos según índices de Barthel, Lawton y baremo de Ley de Dependencia. Rev Soc Esp Enferm Nefrol 2009;12(2):28-34.

4. Andreu Periz L, Moreno Arroyo MC. Perfil y realidad social de los cuidadores principales de pacien-

tes dependientes tratados con hemodiálisis. Nursing (Ed Esp) 2009;27(4):54-65.

5. Teixidó J, Tarrats L, Arias N, Cosculluela A. Cuestionario de sobrecarga de cuidadores de pacientes de diálisis peritoneal. Nefrología 2006;26(1):74-86.

6. Romero-Massa E, Rodríguez-Castilla J, Pereira-Díaz B. Sobrecarga y calidad de vida percibida en cuidadores familiares de pacientes renales. Rev Cubana Enferm. 2015 [citado 02 Dic 2016];31(4):[aprox. 22 p.]. Disponible en: http://www.revenfermeria.sld. cu/index.php/enf/article/view/299/134

7. Romero E, Maccausland Y, Solórzano L. El cuidador familiar del paciente renal y su calidad de vida, Cartagena (Colombia). Rev Científica Salud Uninorte.2006;30(2):146-57.

8. Moreiras-Plaza M. De dónde venimos y adónde vamos en diálisis peritoneal: identificando barreras y estrategias de futuro. Nefrología. 2014;34(6):756-67.

9. Dixon-Woods M, Agarwal S, Jones D, Young B, Sutton $A$. Synthesising qualitative and quantitative evidence: a review of possible methods. J Health Serv Res Policy. 2005;10(1):45-53.

10. Sanz-Valero J, Veiga de Cabo J, Rojo-Alonso C, D’Agostino MJ, Wanden-Berghe C, Espulgues Pellicer JX, et al. Los filtros metodológicos: aplicación a la búsqueda bibliográfica en la medicina del trabajo española. Med Segur Trab 2008; 54(211):75-83.

11. Bobenrieth Astete MA. Lectura crítica de artículos originales en salud. Med Fam. 2001;2:81-90.

12. Cáceres P. Análisis cualitativo de contenido: una alternativa metodológica alcanzable. Psicoperspectivas $2003 ; 2: 53-82$.

13. Castaño C, Hurtado L, Perea D, Sánchez A. Calidad de vida relacionada con la salud de los pacientes y sus cuidadores en una unidad renal de la ciudad de Cali en 2013. Cienc y salud. 2013;2(8):29-35.

14. Martín RA. Estudio sobre percepción de la seguridad clínica de pacientes en diálisis. Rev Mex Urol. 2014;74(5):269-74. 
15. Arimatea Branco JM, Luz Lisboa MT. Tratamento com diálise peritoneal: a prática do autocuidado no contexto familiar. Rev Enferm UERJ. 2015;23(3):344-49.

16. Chan R, Brooks R, Gallagher M, Erlich J, Snelling $\mathrm{P}$, Chow J, et al. Measuring kidney disease-related loss in samples of predialysis and dialysis patients: validating the kidney disease loss scale. Clin J Am Soc Nephrol. 2010;5(7):1249-54.

17. Suri RS, Larive B, Hall Y, Kimmel PL, Kliger AS, Levin N, et al. Effects of Frequent Hemodialysis on Perceived Caregiver Burden in the Frequent Hemodialysis Network Trials. Clin J Am Soc Nephrol. 2014;9(5):936-42.

18. Vázquez $M$, Bolaños $L$, Alonso R. Predictores psicológicos de la calidad de vida relacionada con la salud en pacientes en tratamiento de diálisis peritoneal. Nefrologia 2011;31(1):97-106.

19. Abrahão SS, Ricas J, Andrade DF, Pompeu FC, Chamahum L, Araújo TM, et al. Estudo descritivo sobre a prática da diálise peritoneal em domicílio. J Bras Nefrol. 2010;32(1):45-50.

20. López León D, Rodríguez Calderón L, Carreño Moreno S, Cuenca I, Chaparro Díaz L. Cuidadores de pacientes en diálisis peritoneal: experiencia de participar en un programa de habilidad de cuidado. Enferm Nefrol 2015;18(3):189-95.

21. Ulloa Varela J, Rodríguez Vidal M, Cruz Pedreros M. Perfil biosociodemográfico y valoración de necesidades en pacientes en diálisis peritoneal. Cienc Enferm 2014;20(1):89-95.

22. Arechabala MC, Catoni MI, Palma E, Barrios S. Depresión y autopercepción de la carga del cuidado en pacientes en hemodiálisis y sus cuidadores. Rev Panam Salud Pública. 2011;30(1):74-9.

23. Arenas Jiménez $M$, Reig-Ferrer $A$, Álvarez-Ude F, Malek T, Moledous A, Gil MT, et al. Optimismo disposicional en pacientes en hemodiálisis y su influencia en el curso de la enfermedad. Nefrología 2011;31(2):199-205.

24. Abrahão SS, Ricas J, Andrade DF, Pompeu FC, Chamahum L, Araújo TM, et al. Dificuldades vivenciadas pela família e pela criança/adolescente com doença renal crônica. J Bras Nefrol. 2010;32(1):18-22.

25. Sadala MLA, Bruzos GA de S, Pereira ER, Bucuvic EM. Patients' experiences of peritoneal dialysis at home: a phenomenological approach. Rev Lat Am Enfermagem. 2012;20(1):68-75.

26. Cesar ED, Beuter M, Brondani CM, Pauletto MR, Timm AMB, Jacobi CS. A diálise peritoneal na vivência de familiares cuidadores. Rev RENE. 2000;14(3):541-8.

27. Villarreal-Ríos E, Cárdenas-Maldonado C, Vargas-Daza ER, Galicia-Rodríguez L, Martínez-González L, Baca-Baca R. Institutional and familial cost of patients in continuous ambulatory peritoneal dialysis. Rev Assoc Med Bras. 2014;60(4):335-41.

28. Julián-Mauro JC, Molinuevo-Tobalina JÁ, Sánchez-González JC. La situación laboral del paciente con enfermedad renal crónica en función del tratamiento sustitutivo renal. Nefrología 2012;32(4):439-45.

29. Kiliś-Pstrusińska K, Wasilewska A, Medyńska A, Bałasz-Chmielewska I, Grenda R, Kluska-Jóźwiak A, et al. Psychosocial aspects of children and families of children treated with automated peritoneal dialysis. Pediatr Nephrol. 2013;28(11):2157-67.

30. Pastor JL, Julián JC. Claves del proceso de información y elección de modalidad de diálisis en pacientes con insuficiencia renal crónica. Nefrologia 2010;1(Supl Ext 1):S15-20.

31. Sánchez González JC, Martínez Martínez C, Bethencourt Fernández D, Pablos López M. Valoración de los conocimientos que tienen los pacientes en hemodiálisis acerca de su tratamiento. Enferm Nefrol 2015;18(1):23-30.

32. De las Heras Mayoral MT, Martínez Rincón C. Conocimiento y percepción nutricional en diálisis: su influencia en la transgresión y adherencia: estudio inicial. Nutr Hosp. 2014 Sep 12;31(3):1366-75.

33. Peláez Requejo $B$, Fernández Pérez $M$, Núñez Moral M, González Díaz I, Méndez González A, Quintana Fernández A. Evaluación de los conocimientos prácticos de los pacientes pre- 
valentes en diálisis peritoneal. Enferm Nefrol 2013;16(3):179-84.

34. Burgos Jiménez $E$, Meléndez Balderrama $M A$, Meza Coronado E, Agramón Cota KG, Pereyra Hernández MC, Martínez Menchaca NL. Impacto de una intervención dirigida a incrementar el conocimiento de la enfermedad renal sobre el inicio oportuno de la terapia sustitutiva. Rev Soc Esp Enferm Nefrol2011;14(4):236-41.

35. Carrillo Algarra AJ. Análisis de la capacidad de autocuidados en pacientes en diálisis peritoneal. Enferm Nefrol 2015;18(1):31-40.

36. Neu AM, Richardson T, Lawlor J, Stuart J, Newland $\mathrm{J}$, McAfee $\mathrm{N}$, et al. Implementation of standardized follow-up care significantly reduces peritonitis in children on chronic peritoneal dialysis. Kidney Int. 2016;89(6):1346-54.

37. Gunasekara WD, Ng KH, Chan $\mathrm{YH}$, Aragon $\mathrm{E}$, Foong PP, Lau YW, et al. Specialist pediatric dialysis nursing improves outcomes in children on chronic peritoneal dialysis. Pediatr Nephrol. 2010;25(10):2141-7.

38. Bordin G, Casati M, Sicolo N, Zuccherato N, Eduati V. Patient education in peritoneal dialysis: an observational study in Italy. J Ren Care 2007;33(4):165-71.

39. Gardulf A, Palsson M, Nicolay U. Education for dialysis patients lowers long-term phosphate levels and maintains health-related quality of life. Clin Nephrol 2011;75(4):319-27.

40. Machowska A, Alscher MD, Reddy Vanga S, Koch $M$, Aarup M, Qureshi A, et al. Factors influencing access to education, decision making, and receipt of preferred dialysis modality in unplanned dialysis start patients. Patient Prefer Adherence. 2016;10:2229-37.
41. Robinski M, Mau W, Wienke A, Girndt M. Shared decision-making in chronic kidney disease: A retrospection of recently initiated dialysis patients in Germany. Patient Educ Couns. 2016;99(4):56270.

42. Cohen LM, Ruthazer R, Moss AH, Germain MJ. Predicting six-month mortality for patients who are on maintenance hemodialysis. Clin J Am Soc Nephrol. 2010;5(1):72-9.

43. Moss AH. Revised dialysis clinical practice guideline promotes more informed decision-making. Clin J Am Soc Nephrol. 2010;5(12):2380-93.

44. Janssen DJA, Spruit MA, Schols JMGA, van der Sande FM, Frenken LA, Wouters EFM. Insight into advance care planning for patients on dialysis. $\mathrm{J}$ Pain Symptom Manage. 2013;45(1):104-13.

45. De Rosenroll A, Higuchi KS, Dutton KS, Murray $M A$, Stacey D. Perspectives of significant others in dialysis modality decision-making: a qualitative study. CANNT J.;23(4):17-24.

46. Allen D, Wainwright $M$, Hutchinson T. "Non-compliance" as illness management: Hemodialysis patients' descriptions of adversarial patient-clinician interactions. Soc Sci Med. 2011;73(1):129-34.

47. Walker RC, Howard K, Morton RL, Palmer SC, Marshall MR, Tong A. Patient and caregiver values, beliefs and experiences when considering home dialysis as a treatment option: a semi-structured interview study. Nephrol Dial Transplant. 2016;31(1):133-41.

48. Licht-Ardila M. Rol del profesional de Enfermería en terapias de reemplazo renal, diálisis peritoneal. MedUNAB. 2014;17(2):107-13.

Este artículo se distribuye bajo una Licencia Creative Commons Atribución-NoComercial 4.0 Internacional. https://creativecommons.org/licenses/by-nc/4.0/

Open Access (c) (;) (8) 